\title{
Diferencias en la imagen pública de la ciudad de A Coruña en función del tiempo de permanencia en la ciudad
}

\section{Differences in the public image of the city of A Coruña depending on the time spent in the city}

\author{
María Ángeles González, Jesús A. Dopico y Diego Campos-Juanatey \\ Universidade da Coruña
}

\begin{abstract}
Resumen
En este trabajo se analizaron las diferencias en la imagen pública de la ciudad de A Coruña (Spain), entre las personas que llevaban menos de un año en la ciudad, y las que llevaban más de un año residiendo en ella. Se aplicó el cuestionario utilizado por Boira (1992), con algunas modificaciones, a 130 de estudiantes de la Facultad de Ciencias de Educación de la ciudad. La Torre de Hércules y la Plaza de Pontevedra son elementos representativos para todos ellos. Sin embargo, cuando al grupo que menos tiempo llevaba en la ciudad se le pidió que citara las cinco primeras cosas que recordaba de la ciudad, señaló con más frecuencia las playas de Riazor y Orzán que la Torre de Hércules, y sólo este grupo citó el centro comercial Marineda City entre esos primeros lugares recordados espontáneamente.
\end{abstract}

Palabras clave: Imagen pública, ciudad, familiaridad, cuestionario.

\begin{abstract}
This work aimed to analyze the differences in the image of the city of A Coruña (Spain), among people with less than a year living in the city, and those who took more than a year living in it. To this end, the questionnaire used by Boira (1992), with some modifications, was applied to a sample of 130 students from the Faculty of Education of the city. The Hercules Tower and the Pontevedra Square are representative elements for all of them. However, when participants with less stay in the city had to cite the first five things they remembered from the city, noted in a higher percentage beaches Riazor and Orzán than the Hercules Tower. Only in this group, one of the five places spontaneously remembered more often is the Marineda City shopping center.

Keywords: Public image, city, familiarity, questionnaire.
\end{abstract}

Kevin Lynch, arquitecto norteamericano interesado en el aspecto visual de las ciudades, analizó a finales de los años 50 las zonas centrales de tres ciudades de Estados Unidos (Boston, Nueva Jersey y Los Ángeles), llevando a cabo prolongadas entrevistas con una pequeña muestra de residentes. Los datos obtenidos le permitieron identificar los contenidos de las imágenes de la ciudad en cinco tipos de elementos: Sendas (conductos que conectan distintas partes de la ciudad), bordes (elementos lineales, referencias laterales) barrios (secciones de la ciudad reconocibles), nodos (puntos estratégicos de la ciudad en los que el individuo puede entrar) e hitos (por lo general, objetos físicos bien definidos) (Lynch, 1960/2013).
Según Lynch, una ciudad genera una imagen clara si estos elementos se pueden identificar y organizar fácilmente, de forma que actúen como marco de referencia para desplazarse eficaz y cómodamente por ella. Con la publicación del libro en el que presentaba los resultados de su análisis y su propuesta de elementos de la ciudad, La imagen de la ciudad (Lynch, 1960/2013), popularizó, fundamentalmente entre geógrafos, psicólogos y arquitectos, el estudio del mapa cognitivo de las ciudades (Carreiras, 1992). Este término había sido utilizado previamente en alusión al mapa mental que las ratas parecen usar cuando aprender rutas en laberintos (Tolman, 1948).

Los "mapas" o modelos que construimos de un ambiente por medio de la experiencia, se han denominado también, además de mapa cognitivo, “representación espacial”, “imagen ambiental”, “representación topográfica”, “esquema topográfico”, "imagen espacial”, "mapa mental”, "esquema espacial”, etc., sin embargo, el término más utilizado es el de "mapa cognitivo” (Aragonés y Amérigo, 2010; Lázaro Ruiz, 2000).

Entre los métodos existentes para acercarse al estudio de los mapas cognitivos, el más utilizado ha sido la entrevista, a partir de preguntas abiertas o cerradas, y la elaboración de dibujos y representaciones gráficas del espacio (Boira, 1992), tal y como hizo Lynch en el estudio ya citado (Lynch, 1960/2013).

De las distintas variables personales que han sido analizadas por su posible influencia en la naturaleza y precisión de los mapas cognitivos (sexo, edad, nivel de instrucción, medio de desplazamiento habitual...), la que parece influir de una manera más clara es la familiaridad con la ciudad, medida con frecuencia de forma indirecta a través de los años que una persona pasa viviendo en ella (Carreiras, 1992).

Se ha encontrado que el tiempo de permanencia en una ciudad tiene un efecto positivo sobre algunos aspectos del conocimiento ambiental, como el reconocimiento y recuerdo de hitos y otros elementos urbanos o la estimación correcta de distancias en la ciudad (Boira, 1992; Campos-Juanatey, Pérez-Fabello y Tarrío, en prensa-a; en prensa-b; Dopico, González y Campos-Juanatey, en prensa-a; en prensa-b; Evans, Marrero y Butler, 1981; Golledge y Spector, 1978).

Golledge y Spector (1978) encontraron que cuanto más tiempo se pasa viviendo en una ciudad, menos errores se comenten en la estimación de las distancias. Evans et al. 
(1981) compararon los dibujos de personas que llevaban una semana viviendo en la ciudad, con los que llevaban un año, y confirmaron que las personas que llevaban más tiempo en la ciudad, recordaban más sendas e hitos que los que llevaban en la ciudad una semana.

Campos-Juanatey et al. (en prensa-a) encontraron que las personas que llevaban viviendo en la ciudad de Pontevedra más de un año, tenían un mayor recuerdo de edificios singulares y plazas importantes de la ciudad, que los que llevaban viviendo allí menos de un año. Dopico et al. (en prensa-a) obtuvieron un resultado similar en la ciudad de A Coruña, al comparar estudiantes que llevaban en la ciudad más de 10 años con los que llevaban menos de 10 años. También se ha encontrado que el conocimiento de calles del centro histórico de ambas ciudades, medida a través del recuerdo de sus nombres, es superior en los estudiantes que llevan residiendo más tiempo en ellas (Campos-Juanatey et al., en prensa-b; Dopico et al., en prensa-b).

Boira (1992) llevó a cabo un estudio sobre el mapa cognitivo de los habitantes de Valencia, dividiendo a los participantes en el estudio en tres grupos: los nacidos en Valencia, los que llevaban en ella más de 10 años, y los que llevaban allí menos de un año, y encontró menor propensión a emitir juicios críticos negativos en los que llevaban más tiempo residiendo en la ciudad.

En el presente estudio intentamos averiguar si la imagen que tienen los alumnos de la Facultad de Ciencias de la Educación de la ciudad de A Coruña es diferente en función de que lleven más o menos tiempo residiendo en la ciudad.

\section{Método}

\section{Participantes}

En este estudio participaron 130 estudiantes del primer curso de los Grados de Educación Social y Logopedia de la Facultad de Ciencias de la Educación de A Coruña, 32 hombres y 98 mujeres, con una media de edad de 19.92 años y una desviación típica de 2.49 .

\section{Material}

Se utilizó el cuestionario utilizado por Boira (1992), con algunas modificaciones.

\section{Procedimiento}

Se le entregó el cuestionario al alumnado de primer curso de los grados de Educación Social y de Logopedia, en sus respectivas clases, para que lo cumplimentaran sin límite de tiempo. Posteriormente, se codificaron las respuestas a los diferentes ítems. Para este estudio, de todas las preguntas del cuestionario, en su mayoría preguntas abiertas, se analizó la información referida a la evaluación global de la ciudad, a los lugares que se consideran más céntricos y representativos, y a las preferencias residenciales. Esta información corresponde a los ítems 1, 2, 3, 9, 10, 11 y 15 del cuestionario modificado, al igual que en el cuestionario de Boira (1992).
Para el análisis de las respuestas del cuestionario se dividió en dos grupos a los participantes en función del tiempo de "permanencia en la ciudad". Si los alumnos llevaban un año o menos viviendo en la ciudad de A Coruña, se consideraban de “baja permanencia”, y si llevaban más de un año, se consideraba "alta permanencia”. Esta fue la división empleada por tratarse de estudiantes de primer curso de la Facultad de Ciencias de la Educación, por lo que para muchos éste era su primer año residiendo en la ciudad. Del total de participantes, 57 manifestaron que llevaban un año o menos viviendo en A Coruña, y 73 llevaban más de un año.

\section{Resultados}

La finalidad del estudio era averiguar si existían diferencias en la imagen pública de la ciudad de A Coruña, entre el alumnado de primer curso de los grados de Educación Social y Logopedia de esta ciudad que llevaban una año o menos de un año en la ciudad, y el que llevaba más de un año residiendo en ella. A partir de la codificación de las respuestas de cada uno de estos grupos por separado (en los ítems de la escala que interesaban para este estudio), se investigó el porcentaje de citas recibidas por los lugares o elementos indicados.

La primera información que se obtuvo fue la relacionada con la evaluación global que los encuestados hacían de la ciudad, en respuesta al primer ítem: “¿Cuánto diría que le gusta la ciudad de A Coruña?“. Los porcentajes obtenidos en las diferentes posibilidades de respuesta que ofrecía este ítem, se encuentran en la Figura 1.

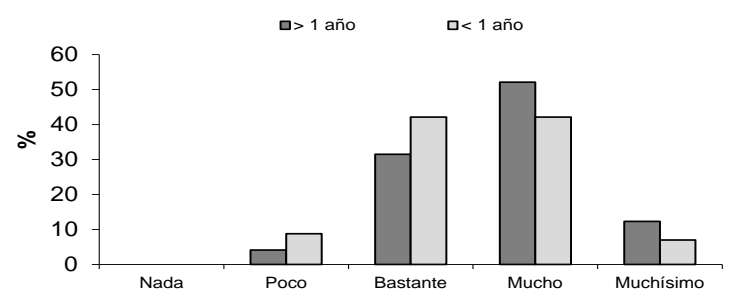

Figura 1. Evaluación global de la ciudad en función del tiempo de permanencia en la ciudad. Respuestas a la pregunta ¿Cuánto diría que le gusta la ciudad de A Coruña?

La mayoría de los participantes de ambos grupos valora la ciudad de forma positiva. Los mayores porcentajes en este ítem se agrupan en las respuestas "bastante" (> 1año = 31,5\%; < 1año= 42,1\%) y "mucho" $(>1$ año $=52,1 \%$; $<1$ año $=42,1 \%)$, frente a un escaso $4,1 \%$ de estudiantes del grupo de alta permanencia, y $8,8 \%$ del de baja permanencia, que afirman que la ciudad les gusta "poco". Ningún participante ha seleccionado "nada" como respuesta a esta pregunta.

A continuación, a los estudiantes se les pedía que enumerasen los cinco lugares de la ciudad de A Coruña que espontáneamente les vinieran a la mente. Los resultados de ambos grupos en esta pregunta abierta, 
ordenados por porcentaje de citas recibidas, se encuentran en la Tabla 1a y en la Tabla 1b. Dado que los porcentajes aparecen en las tablas, por una cuestión de espacio limitaremos la explicación a los resultados más representativos.

Tabla 1(a).

Lugares de la ciudad de A Coruña que espontáneamente vienen a la mente con mayor frecuencia (Ordenados de mayor a menor por porcentaje de veces que han sido citados) (alta permanencia en la ciudad)

\begin{tabular}{clc}
\hline Orden & Nombre del lugar & Porcentaje \\
\hline 1 & Torre de Hércules & 69.86 \\
2 & Monte de San Pedro & 50.68 \\
3 & Riazor/Playa de Riazor-Orzán & 34.25 \\
4 & Calle Real & 32.88 \\
5 & Plaza de María Pita & 19.18 \\
\hline
\end{tabular}

Tabla 1(b)

Lugares de la ciudad de A Coruña que espontáneamente vienen a la mente con mayor frecuencia (Ordenados de mayor a menor por porcentaje de veces que han sido citados) (baja permanencia en la ciudad)

\begin{tabular}{clc}
\hline Orden & Nombre del lugar & Porcentaje \\
\hline 1 & Riazor/Playa de Riazor-Orzán & 70.18 \\
2 & Torre de Hércules & 66.67 \\
3 & Marineda City & 36.84 \\
4 & Plaza de María Pita & 29.82 \\
5 & Cuatro Caminos & 26.32 \\
\hline
\end{tabular}

En esta pregunta abierta, el mayor porcentaje de citas del grupo de alta permanencia ha sido para la Torre de Hércules (69.86\%). En el grupo de baja permanencia, el mayor porcentaje de citas en este ítem ha sido para las playas de Riazor y Orzán (70.18\%). Ambos lugares aparecen en la selección de los dos grupos, aunque con diferentes porcentajes. También la Plaza de María Pita está entre los cinco lugares recordados espontáneamente con más frecuencia por los dos grupos. Sin embargo, Marineda y Cuatro Caminos son dos lugares que sólo cita con frecuencia el grupo de baja permanencia, y la Calle Real y el Monte de San Pedro, el de alta permanencia.

A continuación, en la Tabla 2a y la Tabla 2b, se muestran las respuestas de los encuestados al ítem 3 del cuestionario: "Piense en las diferentes zonas y barrios de la ciudad de A Coruña. ¿Dónde le gustaría vivir si fuera enteramente libre de elegir?”.

Tabla 2(a).

Zona o barrio de la ciudad de A Coruña elegida para vivir (Ordenados de mayor a menor por porcentaje de veces que han sido citados) (alta permanencia en la ciudad)

\begin{tabular}{clc}
\hline Orden & Nombre del lugar & Porcentaje \\
\hline 1 & Riazor/Playa de Riazor-Orzán & 15.07 \\
2 & Monte Alto & 10.96 \\
3 & Ciudad Vieja & 9.59 \\
4 & Ciudad Jardín & 8.22 \\
5 & Plaza de Pontevedra/Rosales & 6.85 \\
\hline
\end{tabular}

Tabla 2(b).

Zona o barrio de la ciudad de A Coruña elegida para vivir (Ordenados de mayor a menor por porcentaje de veces que han sido citados) (baja permanencia en la ciudad)

\begin{tabular}{clc}
\hline Orden & Nombre del lugar & Porcentaje \\
\hline 1 & Riazor/Playa de Riazor-Orzán & 24.56 \\
2 & Plaza de Pontevedra & 21.05 \\
3 & Ronda de Outeiro & 8.77 \\
4 & Cuatro Caminos & 5.26 \\
5 & C/ Real & 5.26 \\
\hline
\end{tabular}

En las respuestas a este ítem se ha encontrado bastante dispersión. Ninguno de los lugares que aparecen en la tabla por haber sido los que más citas de su grupo han obtenido, aglutina un número de ellas superior al $25 \%$. En ambos grupos, la zona elegida para vivir por un mayor porcentaje de encuestados ha sido el entorno de las playas (Riazor y Orzán).

En la Tabla 3a y en la Tabla 3b aparecen las cinco calles o plazas más importantes de la ciudad, en función del número de veces que han sido citadas como respuesta al ítem 9: ¿Cuáles son las cinco calles o plazas más importantes de la ciudad de A Coruña?

Tabla 3(a).

Calles o plazas más importantes de la ciudad (Ordenados de mayor a menor por porcentaje de veces que han sido citados) (alta permanencia en la ciudad)

\begin{tabular}{clc}
\hline Orden & Nombre del lugar & Porcentaje \\
\hline 1 & Plaza de Pontevedra & 82.19 \\
2 & Calle Real & 72.60 \\
3 & Plaza de María Pita & 69.86 \\
4 & Plaza de Lugo & 36.99 \\
5 & Plaza de España & 24.66 \\
\hline
\end{tabular}

Tabla 3(b).

Calles o plazas más importantes de la ciudad (Ordenados de mayor a menor por porcentaje de veces que han sido citados) (baja permanencia en la ciudad)

\begin{tabular}{clc}
\hline Orden & Nombre del lugar & Porcentaje \\
\hline 1 & Plaza de Pontevedra & 80.70 \\
2 & Plaza de María Pita & 63.16 \\
3 & Calle Real & 43.86 \\
4 & Plaza de Lugo & 35.09 \\
5 & Cuatro Caminos & 26.32 \\
\hline
\end{tabular}

La Plaza de Pontevedra aparece citada como una de las plazas más importantes de la ciudad por un porcentaje muy elevado de encuestados de los dos grupos ( $>1$ año: 82,19\%; < 1 año: 80,70\%). Entre los cinco lugares con más citas en esta pregunta sólo ha habido uno diferente en cada grupo. Los estudiantes que llevaban en la ciudad más de un año, seleccionaron con uno de los cinco mayores porcentajes la Plaza de España, mientras que los 
de baja permanencia seleccionaron Cuatro Caminos. La Plaza de María Pita, la Plaza de Lugo y la Calle Real son los otros tres lugares más citados por ambos grupos.

En la Tabla 4a y en la Tabla 4b se muestran los cinco lugares que han sido señalados en otra pregunta abierta como los principales puntos de reunión o encuentro de los residentes de la ciudad.

Tabla 4 (a)

Principales puntos de reunión o encuentro de los residentes de la ciudad (Ordenados de mayor a menor por porcentaje de veces que han sido citados) (alta permanencia en la ciudad)

\begin{tabular}{clc}
\hline Orden & Nombre del lugar & Porcentaje \\
\hline 1 & Plaza de Pontevedra & 56.16 \\
2 & Calle Real & 42.47 \\
3 & Obelisco & 41.10 \\
4 & Plaza de María Pita & 39.73 \\
5 & Jardines de Méndez Núñez & 38.36 \\
\hline
\end{tabular}

Tabla 4 (b)

Principales puntos de reunión o encuentro de los residentes de la ciudad (Ordenados de mayor a menor por porcentaje de veces que han sido citados) (baja permanencia en la ciudad)

\begin{tabular}{clc}
\hline Orden & Nombre del lugar & Porcentaje \\
\hline 1 & Plaza de Pontevedra & 57.89 \\
2 & Jardines de Méndez Núñez & 47.37 \\
3 & Marineda City & 35.09 \\
4 & Obelisco & 33.33 \\
5 & Cuatro Caminos & 28.07 \\
\hline
\end{tabular}

La Plaza de Pontevedra es el lugar que los participantes de alta y de baja permanencia en la ciudad han señalado como principal punto de reunión o encuentro de los residentes (56.16\% y 57.89\%, respectivamente). Otros lugares destacados por ambos grupos son el Obelisco y los Jardines de Méndez Núñez, aunque con diferentes porcentajes de citas. La Plaza de María Pita y la Calle Real son dos de los cinco lugares más citados sólo por el grupo de alta permanencia, mientras que Marineda y Cuatro Caminos sólo son citados por el grupo que lleva menos tiempo residiendo en la ciudad.

Para saber qué lugares consideran los participantes del estudio como lo más representativo de la ciudad, se les preguntó por los cinco lugares o cosas de la ciudad de A Coruña que señalarían a un extranjero que la visitara por primera vez. En la Tabla 5a y la Tabla 5b se presentan los lugares más citados como respuesta a este ítem.

Tabla 5(a).

Lugares o cosas de la ciudad que señalarían a quien la visitara por primera vez (Ordenados de mayor a menor por porcentaje de veces que han sido citados) (alta permanencia en la ciudad)

\begin{tabular}{ccc}
\hline Orden & Nombre del lugar & Porcentaje \\
\hline 1 & Torre de Hércules & 94.52 \\
2 & Monte de San Pedro & 57.53
\end{tabular}

\begin{tabular}{lll}
3 & Ciudad Vieja & 34.25 \\
4 & Paseo Marítimo & 26.03 \\
5 & Plaza de María Pita & 26.03 \\
\hline
\end{tabular}

Tabla 5(b).

Lugares o cosas de la ciudad que señalarían a quien la visitara por primera vez (Ordenados de mayor a menor por porcentaje de veces que han sido citados) (baja permanencia en la ciudad)

\begin{tabular}{ccc}
\hline Orden & Nombre del lugar & Porcentaje \\
\hline 1 & Torre de Hércules & 89.47 \\
2 & Playas Riazor-Orzán & 56.14 \\
3 & Monte de San Pedro & 45.61 \\
4 & Casa de los peces & 36.84 \\
5 & Plaza de María Pita & 31.58 \\
\hline
\end{tabular}

Prácticamente la totalidad de los encuestados señalan a la Torre de Hércules como lo primero que enseñarían a alguien que visitara la ciudad por primera vez (en torno al $90 \%$ en ambos grupos), con bastante diferencia -en porcentaje de elecciones- de los siguientes lugares señalados, que son, para el grupo de alta permanencia, el Monte de San Pedro (57,53\%), y para el de baja permanencia, las Playas de Riazor y Orzán (56,14\%). En ambos grupos el Monte de San Pedro y la Plaza de María Pita están entre los cinco lugares con mayores porcentajes de citas para esta pregunta.

En el último ítem analizado en este estudio se preguntaba: “¿Dónde tiene usted la impresión de hallarse en pleno corazón de la ciudad de A Coruña? (pregunta cerrada; alternativas de respuesta: En la Plaza de Azcárraga, en la Plaza de María Pita, en la Plaza de Pontevedra, en otro lugar)”. Las respuestas aparecen en la Figura 2.

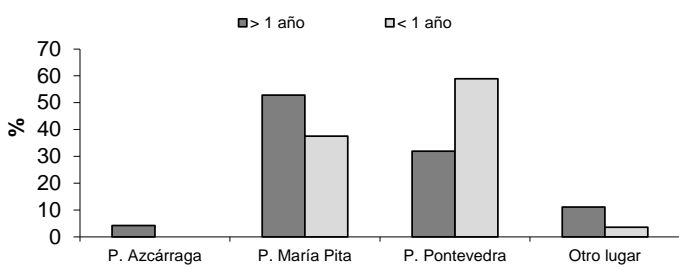

Figura 2. Lugar destacado como "Corazón de la ciudad" de A Coruña, en función del tiempo de permanencia en la ciudad.

Aunque la mayoría de elecciones ha correspondido a la Plaza de María Pita y a la Plaza de Pontevedra, el lugar que ha recibido un mayor número de citas como corazón de la ciudad varía dependiendo del grupo de que se trate. El porcentaje más alto de citas en el grupo de alta permanencia ha sido para la Plaza de María Pita (52,8\%), mientras que el grupo de baja permanencia considera como corazón de la ciudad mayoritariamente a la Plaza de Pontevedra (seleccionada por el 58,9\% de participantes). Ningún encuestado del grupo de baja permanencia seleccionó la Plaza de Azcárraga como corazón de la ciudad. 


\section{Discusión}

Este trabajo tenía como objetivo analizar si existían diferencias en la imagen de la ciudad de A Coruña, entre las personas que llevaban menos de un año viviendo en la ciudad, y las que llevaban más de un año residiendo en ella. Casi todos los participantes, independientemente del tiempo transcurrido desde su llegada a la ciudad, citaron a la Torre de Hércules entre las cinco cosas que enseñarían a alguien que visitara la ciudad por primera vez. Este es un dato más sobre el papel que juega este elemento en la configuración de la imagen de la ciudad, algo ya conocido y en la línea de los resultados de otros estudios sobre esta ciudad (Dopico et al., en prensa-a; González, Dopico y Campos-Juanatey, en prensa).

Sin embargo, cuando a los participantes con menor permanencia en la ciudad se les pedía que citaran cinco lugares que recordaban de la ciudad, estos señalaron en mayor porcentaje las playas de Riazor y Orzán que la Torre de Hércules (aunque ésta ocupaba el siguiente lugar en esa clasificación).

Un resultado a destacar es que en el grupo de estudiantes con menor permanencia en la ciudad, otro de los cinco lugares recordados de forma espontánea con más frecuencia es el complejo comercial Marineda City, al que también consideran como uno de los principales puntos de reunión o encuentro. Bernal Santa Olalla (2003) destacó la importancia que los jóvenes dan a los centros comerciales como un cambio significativo y real en los hábitos de ocio en esta población (que en su informe eran estudiantes de secundaria).

El hecho de que con este lugar se dé esta diferencia entre los estudiantes con menor y mayor permanencia en la ciudad es un dato interesante, acorde con la idea de que la ciudad es un espacio vivo que cambia con el tiempo. Este gran centro comercial abrió sus puertas hace cuatro años. Para quienes llegaron a la ciudad hace un año o menos de un año ha sido ya un elemento a considerar cuando estaban formando su imagen de la ciudad, mientras que, probablemente, el proceso de incorporación de este elemento a la imagen de la ciudad será más lento para aquéllos individuos nativos o con una permanencia de más años en la ciudad.

El lugar que más personas del grupo que lleva menos tiempo residiendo en la ciudad perciben como "corazón” de la ciudad es la Plaza de Pontevedra, mientras que en el grupo de mayor permanencia en la ciudad, este lugar lo ocupa la Plaza de María Pita. Sin embargo, todos los encuestados señalaron a la plaza de Pontevedra, importante nodo de comunicaciones de la ciudad, como la plaza más importante de la ciudad, y el principal punto de reunión o encuentro.

En general, y en mayor o menor grado, aparecen en las respuestas de los encuestados todos los elementos señalados por Lynch. Resultaría de interés la confirmación de los resultados que hemos encontrado en este análisis, a través del estudio del dibujo del mapa de la ciudad, asumidas las limitaciones de dicho método (Lázaro Ruíz, 2000). Por último, sería interesante también llevar a cabo una comparación entre los resultados obtenidos a través de la encuesta con estos estudiantes, con la imagen que organismos públicos (información turística, fundamentalmente) y medios de comunicación proyectan de la ciudad, como realizó Boira (1992) en su estudio.

\section{Referencias}

Aragonés, J. I., y Amérigo, M. (2010). Psicología ambiental. Aspectos conceptuales y metodológicos. En J. I. Aragonés y M. Amérigo. Psicología ambiental (pp. 25-55). Madrid: Pirámide.

Bernal Santa Olalla, B. (2003). Los jóvenes y la ciudad. Análisis y valoración del patrimonio urbano en quince ciudades históricas. En L. López, C. E. Relea, y J. Somoza (Coord.), La ciudad. Nuevos procesos, nuevas respuestas (pp. 373-387). León: Universidad de León.

Boira, J. V. (1992). La ciudad de Valencia y su imagen pública. Valencia: Universidad de Valencia.

Carreiras, M. (1992). Mapas cognitivos y orientación espacial. En J. Mayor y J L. Pinillos (Eds.), Tratado de psicología general (Vol. 4): Memoria y representación (pp. 375-408). Madrid: Alhambra Universidad.

Campos-Juanatey, D., Pérez-Fabello, M. J., y Tarrío, S. (en prensa-a). El mapa cognitivo de los estudiantes de Bellas Artes I: El conocimiento de hitos urbanos. Actas del XIII Congreso Internacional Galego-Portugués de Psicopedagoxía. A Coruña.

Campos-Juanatey, D., Pérez-Fabello, M. J., y Tarrío, S. (en prensa-b). El mapa cognitivo de los estudiantes de Bellas Artes II: El conocimiento del centro histórico de Pontevedra. Actas del XIII Congreso Internacional Galego-Portugués de Psicopedagoxía. A Coruña.

Dopico, J., González, M. A. y Campos-Juanatey, D. (en prensa-a). Influencia de la familiaridad con una ciudad en su conocimiento. Actas del XIII Congreso Internacional Galego-Portugués de Psicopedagoxía. A Coruña.

Dopico, J., González, M. A. y Campos-Juanatey, D. (en prensa-b). ¿Influye la habilidad de rotar imágenes en el conocimiento de una ciudad? Actas del XIII Congreso Internacional Galego-Portugués de Psicopedagoxía. A Coruña.

Evans, G. W., Marrero, D. G., y Butler, P. A. (1981). Environmental learning and cognitive mapping. Environment and Behavior, 13, 83-104.

Golledge, R. G., y Spector, A. N. (1978). Comprehending the urban environment: Theory and practice. Geographycal Analysis, 10, 401-426.

González, M. A., Dopico, J. A. y Campos-Juanatey, D. (en prensa). La imagen pública de la ciudad de A Coruña. Actas del XIII Congreso Internacional Galego-Portugués de Psicopedagoxía. A Coruña.

Lázaro Ruiz, V. (2000). La representación mental del espacio a lo largo de la vida. Zaragoza: Egido Editorial.

Lynch, K. (1960). The image of the city. Cambridge, MA: MIT Press. [Trad. Cast. (2013). La imagen de la ciudad. Barcelona: Gustavo Gili].

Tolman, E. C. (1948). Cognitive maps in rats and men. Psychological Review, 55, 189-208. 